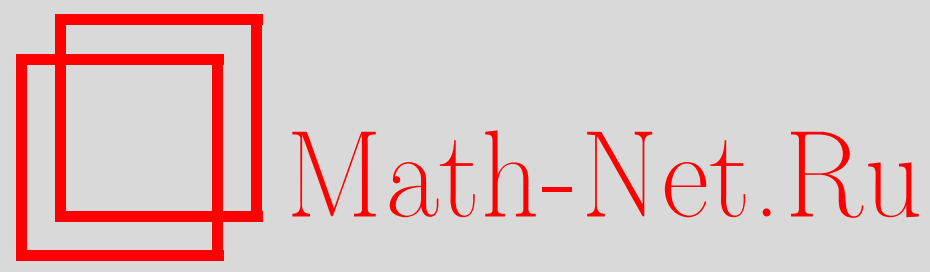

С. Лорд, А. А. Седаев, Ф. А. Сукочев, Следы КоннаДиксмье, сингулярные симметричные функционалы и понятие измеримых по Конну элементов, Матем. заметки, 2005, том 77, выпуск 5, 727-732

DOI: https://doi.org/10.4213/mzm2528

Использование Общероссийского математического портала Math-Net.Ru подразумевает, что вы прочитали и согласны с пользовательским соглашением http://www.mathnet.ru/rus/agreement

Параметры загрузки:

IP : 54.198 .64 .247

26 апреля 2023 г., 18:18:58

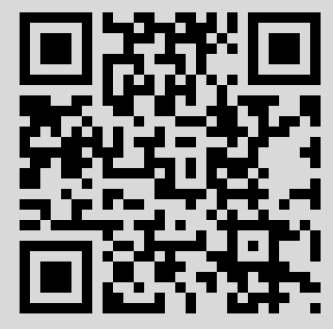




\title{
СЛЕДЫ КОННА-ДИКСМЬЕ, СИНГУЛЯРНЫЕ СИММЕТРИЧНЫЕ ФУНКЦИОНАЛЫ И ПОНЯТИЕ ИЗМЕРИМЫХ ПО КОННУ ЭЛЕМЕНТОВ
}

\author{
С. Лорд, А. А. Седаев, Ф. А. Сукочев
}

Работа продолжает исследование, начатое в [1]-[5]. Показано, что конструкция анормальных следов, примененная в [1], [2], может быть адекватно выражена с помощь конструкции сингулярных симметричных функционалов, разработанной в [4], [5]. Получено полное описание измеримых элементов, на которых все сингулярные симметричные функционалы из некоторого класса принимают одинаковые значения [1]. Этот результат существенно дополняет описание структуры множества измеримых операторов даже для специального случая, рассмотренного в [1]. Для естественных подмножеств множества сингулярных симметричных функционалов получены новые результаты об их нормирующих свойствах.

Библиограффия: 7 названий.

Мы будем оперировать с множеством $\Omega_{\infty}$ вогнутых функций $\psi: \mathbb{R}_{+} \rightarrow \mathbb{R}_{+}$таких, что $\lim _{t \rightarrow 0^{+}} \psi(t)=0$ и $\lim _{t \rightarrow \infty} \psi(t)=\infty$ и с множеством $\mathscr{K}$ неотрицательных возрастающих и непрерывных функций $k$ таких, что $\lim _{t \rightarrow \infty} k(t)=\infty$.

ОПРЕДЕЛЕНИЕ 1 . Пусть $\psi \in \Omega_{\infty}$ и $\kappa \in \mathscr{K}$. Говорят, что

- $\kappa$ обладает свойством $D$ относительно $\psi$ (обозначение: $\kappa \in D(\psi)$ ), если $\psi(\kappa(t)) / t$ есть невозрастающая на $\mathbb{R}_{+}$функция;

- $\kappa$ удовлетворяет условию $R$ относительно $\psi$ (обозначение: $\kappa \in R(\psi)$ ), если

$$
\underset{n \rightarrow \infty}{\mathrm{F}-\lim } \frac{\psi(\kappa(n))}{\psi(\kappa(n+1))}=1
$$

- $\kappa$ есть функиия экспоненииального роста, если $\exists C>0$ такое, что $\kappa(t+C)>$ $2 \kappa(t)$ для всех $t>0$.

Подмножество элементов $R(\psi)$ экспоненциального роста обозначается $R_{\exp }(\psi)$.

Работа первого автора выполнена при поддержке Australian Research Council. Работа второго автора выполнена при поддержке Российского фонда фундаментальных исследований, грант № 02-01-00146, и научно-исследовательской программы "Университеты России", грант УР 04.01.051. 
TeOpema 1. Пусть $\psi \in \Omega_{\infty}$. Тогда

1) $R_{\exp }(\psi)$ не пусто тогда и только тогда, когда

$$
\liminf _{t \rightarrow \infty} \frac{\psi(2 t)}{\psi(t)}=1
$$

2) $R_{\exp }(\psi)$ содержит функцию к такую, что

$$
\lim _{n \rightarrow \infty} \frac{\psi(\kappa(n))}{\psi(\kappa(n+1))}=1
$$

в том и только том случае, если $\lim _{t \rightarrow \infty}(\psi(2 t) / \psi(t))=1$;

3) если $\kappa \in D(\psi)$, то $\kappa \in R(\psi) u$

$$
\lim _{n \rightarrow \infty} \frac{\psi(\kappa(n))}{\psi(\kappa(n+1))}=1 ;
$$

4) обратная функиия $\psi^{-1}$ будет экспоненииального роста тогда и только тогда, когда $\exists C, t_{0}>0$ такие, что $\psi^{\prime}(t) \leqslant C / t$ для всех $t>t_{0}$.

Напомним обозначения из [4]. Пусть $m$ - мера Лебега на $\mathbb{R}_{+}$и $x$ - измеримая, почти всюду конечная на $\mathbb{R}_{+}$функция. Определим для $x$ невозрастающую перестановку.

Пусть $\psi \in \Omega_{\infty}$. Положим

$$
\phi(x)(t):=\frac{1}{\psi(t)} \int_{0}^{t} x^{*}(s) d s, \quad t>0,
$$

и обозначим через $M(\psi)$ пространство Марцинкевича измеримых на $\mathbb{R}_{+}$функций $x$ таких, что

$$
\|x\|_{M(\psi)}:=\sup _{t>0} \phi(x)(t)=\|\phi(x)\|_{\infty}<\infty .
$$

Замыкание $M(\psi) \cap L^{1}\left(\mathbb{R}_{+}\right)$в $M(\psi)$ обозначается $M_{1}(\psi)$. Для любой $\psi \in \Omega_{\infty}$ имеем $M_{1}(\psi) \neq M(\psi)$. Определим на $M(\psi)$ полунорму Рисса $\rho_{1}(\cdot)$ по формуле

$$
\rho_{1}(x):=\inf \left\{\|x-y\|_{M(\psi)} \mid y \in M_{1}(\psi)\right\}=\limsup _{t \rightarrow \infty} \phi(x)(t)
$$

(см. [4, предложение 2.1]). Банахово пространство $\left(M(\psi),\|\cdot\|_{M(\psi)}\right)$ есть пример симметричного (или перестановочно-инвариантного) пространства [6]. Пусть $M_{+}(\psi)$ означает конус положительных функций из $M(\psi)$.

ОПРЕДЕЛЕНИЕ 2. Положительный однородньй функционал $f: M_{+}(\psi) \rightarrow \mathbb{R}_{+}$называется

(i) симметричным, если $f(x) \leqslant f(y)$ для всех $x, y \in M_{+}(\psi)$ таких, что

$$
\int_{0}^{t} x^{*}(s) d s \leqslant \int_{0}^{t} y^{*}(s) d s \quad \forall t \in \mathbb{R}_{+} ;
$$

(ii) сосредоточенным.м на бесконечности и сингулярным, если $f(|x|)=0$ для всех $x \in M_{1}(\psi)$.

Если такой функционал является аддитивным, то он может быть продолжен по линейности до ограниченного линейного положительного функционала на $M(\psi)$. Пусть $M_{+}(\psi)_{\text {sym }, \infty}^{*}$ означает конус таких функционалов $[4, \S 2]$. Не всякое пространство Марцинкевича $M(\psi), \psi \in \Omega_{\infty}$, допускает существование линейных сингулярных симметричных функционалов. Необходимые и достаточные условия были найдены в [3, теорема 3.4] (смотри также теорему 3 и пункт 1) теоремы 1). 
1. Мы обозначаем через $B L(\mathbb{N})$ множество банаховых пределов, т.е. множество линейных инвариантных относительно сдвига положительных функционалов $L^{\prime}$ на пространстве $\ell_{\infty}$ таких, что $L^{\prime}(\mathbf{1})=1$. Аналогично через $B L\left(\mathbb{R}_{+}\right)$мы обозначаем множество всех банаховых пределов $L$, определенных на пространстве $C_{b}\left(\mathbb{R}_{+}\right)$всех ограниченных и непрерывных функций. Заметим, что все банаховы пределы обращаются в нуль на $C_{0}\left(\mathbb{R}_{+}\right)$- подпространстве непрерьвных и стремящихся к нулю функций.

Обозначим через $S C_{b}^{*}\left(\mathbb{R}_{+}\right)$множество всех положительных линейных функционалов $\gamma$ на $C_{b}\left(\mathbb{R}_{+}\right)$таких, что $\gamma(\mathbf{1})=1$ и $\gamma(f)=0$ для всех $f$ из $C_{0}\left(\mathbb{R}_{+}\right)$.

Пусть $C$ означает оператор чезаровского среднего, задаваемый равенством

$$
C(g)(t)=\frac{1}{t} \int_{0}^{t} g(s) d s
$$

для $g \in C_{b}\left(\mathbb{R}_{+}\right)$.

ОПРЕДЕЛЕНИЕ 3. Пусть $\gamma \in S C_{b}^{*}\left(\mathbb{R}_{+}\right)$. Положительный линейньй функционал на $C_{b}\left(\mathbb{R}_{+}\right)$вида $L_{\gamma}:=\gamma \circ C$ мы назовем пределом Чезаро-Банаха. Пусть $C B L\left(\mathbb{R}_{+}\right)$означает множсество пределов Чезаро-Банаха на $C_{b}\left(\mathbb{R}_{+}\right)$.

Легко проверить, что $L_{\gamma}$ инвариантен относительно сдвига и, значит, $C B L\left(\mathbb{R}_{+}\right) \subset$ $B L\left(\mathbb{R}_{+}\right)$.

ОПРЕДЕЛЕНИЕ 4. Пусть $\psi \in \Omega_{\infty}$ и $\kappa \in \mathscr{K}$. Для $x \in M_{+}(\psi)$ и $L^{\prime} \in B L(\mathbb{N})$ определим $f_{L^{\prime}, \kappa}(x):=L^{\prime}\left(\left\{\phi_{\kappa}(x)(n)\right\}_{n=1}^{\infty}\right)$.

В [4] были найдены необходимые и достаточные условия на последовательность $\{\kappa(n)\}_{n=1}^{\infty}$ и функцию $\psi \in \Omega_{\infty}$, при выполнении которых $f_{L^{\prime}, \kappa}$ принадлежит $M_{+}(\psi)_{\mathrm{sym}, \infty}^{*}$ для всех $L^{\prime} \in B L(\mathbb{N})$. Естественно ввести следующее обобщение.

ОПРеДЕЛЕНИЕ 5. Пусть $\psi \in \Omega_{\infty}$ и $\kappa \in \mathscr{K}$. Для $x \in M_{+}(\psi)$ и $L \in B L\left(\mathbb{R}_{+}\right)$определим $f_{L, \kappa}(x):=L\left(\phi_{\kappa}(x)\right)$.

ПРЕДЛОЖЕНИЕ 1. Пусть $\psi \in \Omega_{\infty}, L \in B L\left(\mathbb{R}_{+}\right) u L^{\prime} \in B L(\mathbb{N}) . E c л u \kappa_{1}, \kappa_{2} \in \mathscr{K}$ таковы, что $\kappa_{1}-\kappa_{2}$ ограничена, то $f_{L, \kappa_{1}}(x)=f_{L, \kappa_{2}}(x) u f_{L^{\prime}, \kappa_{1}}(x)=f_{L^{\prime}, \kappa_{2}}(x) \partial \Omega_{\text {я }}$ всех $x \in M_{+}(\psi)$.

ЗАмЕчАнИЕ 1. Предложение 1 вводит понятие эквивалентности для функций $\kappa \in \mathscr{K}$, которое выражается в одинаковости функционалов $f_{L, \kappa}(x)$ на $M_{+}(\psi)$. Из предложения 1 легко следует, что для каждой $\kappa \in \mathscr{K}$ класс эквивалентности $[\kappa]$ содержит строго возрастающую обратимую кусочно-линейную и дифференцируемую функцию такую, что $\kappa(0)=0$. Этот факт будет использован в теореме 4.

Пусть $H$ есть $\mathbb{N}$ или $\mathbb{R}_{+}$. Обобщим понятие почти сходящейся последовательности [7].

ОПРЕДЕЛЕНИЕ 6. Функция $f \in C_{b}(H)$ называется почти сходящейся (на бесконечности) относительно $V \subset B L(H)$, если $L_{1}(f)=L_{2}(f) \forall L_{1}, L_{2} \in V$.

Мы обозначим значение $A:=L(f) \forall L \in V$ через $\mathrm{F}_{V}$-lim $f=A$, сохранив специальное обозначение $\mathrm{F}-\lim f=A$ для $V=B L(H)$.

Tеорема 2. Eсли $\kappa \in R(\psi), \operatorname{mo}\left\{f_{L, \kappa} \mid L \in B L\left(\mathbb{R}_{+}\right)\right\}=\left\{f_{L^{\prime}, \kappa} \mid L^{\prime} \in B L(\mathbb{N})\right\}$. 
СлЕДСтвИЕ 1. Пусть $\psi \in \Omega_{\infty}, \kappa \in R(\psi) u x \in M_{+}(\psi)$. Тогда

$$
\underset{t \rightarrow \infty}{\mathrm{F}-\lim _{\kappa}} \phi_{\kappa}(x)(t)=A
$$

в том и только том случае, когда

$$
\underset{n \rightarrow \infty}{\mathrm{F}-\lim _{\boldsymbol{N}}} \phi_{\kappa}(x)(n)=A \quad \text { для некоторого } A \geqslant 0 .
$$

Tеорема 3. Пусть $\psi \in \Omega_{\infty} u \kappa \in R(\psi)$. Тогда утверждения

(i) $f_{L^{\prime}, \kappa} \in M_{+}(\psi)_{\mathrm{sym}, \infty}^{*} \forall L^{\prime} \in B L(\mathbb{N})$;

(ii) $f_{L, \kappa} \in M_{+}(\psi)_{\mathrm{sym}, \infty}^{*} \forall L \in B L\left(\mathbb{R}_{+}\right)$;

(iii) $\kappa \in R_{\exp }(\psi)$

әквивалентны.

2. Конструкция Конна-Диксмье [1, IV .2] порождает сингулярные симметричные функционалы на $M_{+}(\psi)$ для специальной функции $\psi(t)=\log (1+t)$. Мы обобщим эту конструкцию на произвольные пространства Марцинкевича $M(\psi)$ для $\psi \in \Omega_{\infty}$ и покажем, что построенные таким образом функционалы образуют подкласс функционалов вида $f_{L, \kappa}$, введенных и изученных выше.

Пусть $k: \mathbb{R}_{+} \rightarrow \mathbb{R}_{+}$есть непрерьвная возрастающая кусочно-дифференцируемая функция. Определим

$$
M_{k}(g)(\lambda):=\frac{1}{k(\lambda)} \int_{0}^{\lambda} g(s) d k(s),
$$

где $g \in C_{b}\left(\mathbb{R}_{4}\right)$ и $\lambda>0$.

ОПРЕДЕЛЕниЕ 7 (сравни с определением в [1, IV.2]). Пусть $\psi \in \Omega_{\infty}$ и $k \in \mathscr{K}$ есть кусочно-дифференцируемая функция, $k(0)=0$. Назовем функиионалом Конна-Диксмье на $M_{+}(\psi)$ функционал $\tau_{\gamma, k}(x)=\gamma \circ M_{k}(\phi(x)), x \in M_{+}(\psi)$, где $\gamma \in S C_{b}^{*}\left(\mathbb{R}_{+}\right)$.

Пусть $k \in \mathscr{K}, k(0)=0$. Определим функцию $g_{k}(t):=g(k(t))$ для всех $t>0$ и $g \in C_{b}\left(\mathbb{R}_{+}\right)$. Ясно, что $g \rightarrow g_{k}$ есть *-автоморфизм $C_{b}\left(\mathbb{R}_{+}\right)$.

Пусть $\gamma \in S C_{b}^{*}\left(\mathbb{R}_{+}\right)$. Тогда функционал $\gamma_{k}$ на $C_{b}\left(\mathbb{R}_{+}\right)$, определенный равенством $\gamma_{k}(g):=\gamma\left(g_{k}\right) \forall g \in C_{b}\left(\mathbb{R}_{+}\right)$, также является элементом $S C_{b}^{*}\left(\mathbb{R}_{+}\right)$.

ПреДлОЖЕнИЕ 2. Пусть $k \in \mathscr{K}-$ кусочно-дифференцируемая функиия $и k(0)=0$. Tогда $\gamma \circ M_{k}(g)=\gamma_{k} \circ C\left(g_{k^{-1}}\right)=L_{\gamma_{k}}\left(g_{k^{-1}}\right)$ для всех $g \in C_{b}\left(\mathbb{R}_{+}\right)$, где $L_{\gamma_{k}} \in C B L\left(\mathbb{R}_{+}\right)$ описан в определении 3.

Как следствие, мы получаем следующий идентификационный результат.

Теорема 4. Если $k$ кусочно-дифферениируема и $k(0)=0$, то множество функuионалов Конна-Диксмье, возникающих из функиии $k$, есть

$$
\left\{\tau_{\gamma, k} \mid \gamma \in S C_{b}^{*}\left(\mathbb{R}_{+}\right)\right\}=\left\{f_{L, k^{-1}} \mid L \in C B L\left(\mathbb{R}_{+}\right)\right\} .
$$

ОПРЕДЕЛЕНИЕ 8. Пусть $V \subset B L\left(\mathbb{R}_{4}\right)$. Будем говорить, что $V$ обладает свойством чезаровского предела, если для любого $g \in C_{b}\left(\mathbb{R}_{+}\right),\{a, b\} \subset\{L(g) \mid L \in V\}$, где $a=\lim \inf _{t \rightarrow \infty} C(g)(t)$ и $b=\lim \sup _{t \rightarrow \infty} C(g)(t)$. 
ПрЕДЛОЖенИЕ 3. Множество пределов Чезаро-Банаха $C B L\left(\mathbb{R}_{+}\right)$обладает свойством чезаровского предела.

Определим полунорму, полагая $\|x\|_{\kappa, V}:=\sup \left\{f_{L, \kappa}(|x|) \mid L \in V\right\}$ для $x \in M(\psi)$.

Tеорема 5. Пусть $\psi \in \Omega_{\infty}, \kappa \in D(\psi)$, и пусть $V \subset B L\left(\mathbb{R}_{+}\right)$обладает свойством чезаровского предела. Тогда

$$
\frac{1}{e} \rho_{1}(x) \leqslant\|x\|_{\kappa, V} \leqslant \rho_{1}(x) \quad \forall x \in M(\psi) .
$$

3. Пусть $\psi \in \Omega_{\infty}, \kappa \in \mathscr{K}$ и $V \subset B L\left(\mathbb{R}_{+}\right)$обладает свойством чезаровского предела. Имея семейство $\left\{f_{L, \kappa} \mid L \in V\right\}$ функционалов, заданных на $M_{+}(\psi)$, естественно рассмотреть элементы $x$ из $M_{+}(\psi)$ такие, что

$$
f_{L_{1}, \kappa}(x)=f_{L_{2}, \kappa}(x) \quad \forall L_{1}, L_{2} \in V .
$$

Очевидно, равенство (2) выполняется тогда и только тогда, когда $\phi(x)(t)$ почти сходится на бесконечности относительно $V$ и, значит, $f_{L, \kappa}(x)=\mathrm{F}_{V^{-}} \lim _{t \rightarrow \infty} \phi_{\kappa}(x)(t)$. Однако почти сходимость не является прозрачным и функциональньм условием. Ниже мы рассмотрим другие условия, которые вместе с некоторым предположением на рост $\kappa$ окажутся эквивалентными свойству (2).

ОПРЕДЕЛЕНИЕ 9. Функция $g \in C_{b}\left(\mathbb{R}_{+}\right)$назьвается

(i) сходящейся по Чезаро, если существует $\lim _{t \rightarrow \infty} C(g)(t)$,

(ii) $V$-почти сходящейся на бесконечности, если существует $\mathrm{F}_{V}-\lim _{t \rightarrow \infty} g(t)$,

(iii) сходящейся на бесконечности, если существует $\lim _{t \rightarrow \infty} g(t)$.

Обозначаем через $\mathscr{C}, \mathscr{F} V \mathscr{S}$ множества сходящихся по чезаро, $V$-почти сходящихся и сходящихся на бесконечности функций, соответственно. Поскольку $V$ обладает свойством чезаровского предела, имеем вложения $\mathscr{S} \subset \mathscr{F} V \subset \mathscr{C}$.

ОПРеДЕЛЕнИЕ 10. Пусть $\psi \in \Omega_{\infty}, \kappa \in \mathscr{K}$, и пусть $x \in M_{+}(\psi)$. Следуя Конну [1] будем говорить, что $x$ является $\mathscr{C}_{\kappa}$-измеримым, если $\phi_{\kappa}(x) \in \mathscr{C}, \mathscr{F}_{V, \kappa^{-}}$измеримым, если $\phi_{\kappa}(x) \in \mathscr{F}_{V}$, и $\mathscr{S}$-измеримым (или тауберовым), если $\phi(x) \in \mathscr{S}$.

Tеорема 6. Пусть $\psi \in \Omega_{\infty}, \kappa \in R(\psi)$ и пусть $x \in M_{+}(\psi)$ таков, что функиия

$$
\sigma(t)=t \phi_{\kappa}(x)(t)
$$

не убьвает. Тогда для такого х три определения измеримости, приведенные в определении 10, әквивалентны между собой.

Заметим, что если $\kappa \in D(\psi)$, то условие (3) в теореме 6 выполнено для всех $x \in M(\psi)$. Доказательство теоремы 6 основывается на следующей теореме тауберова типа.

Теорема 7. Пусть $\sigma$ есть неубьвающая положительная на $\mathbb{R}_{+}$функция. Тогда функция $\sigma(t) / t$ сходится на бесконечности в том и только том случае, если она сходится по Чезаро. 
ЗАмЕчАниЕ 2. В [4] было показано, что если

a

$$
\liminf _{t \rightarrow \infty} \psi(2 t) / \psi(t)=1
$$

$$
\limsup _{t \rightarrow \infty} \psi(2 t) / \psi(t)=2,
$$

то существует $x_{0}>0$ из $M(\psi) \backslash M_{1}(\psi)$ такой, что все сингулярные симметричные функционалы, определенные на $M(\psi)$, обращаются в нуль на $x_{0}$. Однако, если бы $\phi(x)(t) \rightarrow 0$ при $t \rightarrow \infty$, то $\rho_{1}\left(x_{0}\right)=0$ и $x_{0} \in M_{1}(\psi)$, что приводит к противоречию. Следовательно, существуют $\psi \in \Omega_{\infty}$ такие, что для любого $\kappa \in \mathscr{K}$, принадлежащего $R_{\exp }(\psi)$, множества $\mathscr{C}_{\kappa}$-измеримых и $\mathscr{S}$-измеримых элементов не идентичны.

В заключение рассмотрим частньй случай $V=C B L\left(\mathbb{R}_{+}\right)$. С учетом отождествления (1) полученные выше результаты применимы к функционалам Конна-Диксмье.

ТеОрема 8. Пусть $\psi \in \Omega_{\infty} u k \in \mathscr{K}, k(0)=0$, является кусочно дифферениируемой функиией такой, что $k^{-1} \in D(\psi)$. Тогда

А) для всех $x \in M(\psi)$

$$
\rho_{1}(x) \simeq \sup \left\{\tau_{\gamma, k}(|x|) \mid \gamma \in S C_{b}^{*}\left(\mathbb{R}_{+}\right)\right\}
$$

В) следующие утверждения әквивалентны:

(i) $x$ является $\mathscr{C}_{k^{-1}}-$ измеримым;

(ii) $\tau_{\gamma, k}(x)$ не зависит от $\gamma \in S C_{b}^{*}\left(\mathbb{R}_{+}\right)$;

(iii) $\tau_{\gamma, k}(x)=\lim _{t \rightarrow \infty} \frac{1}{\psi(t)} \int_{0}^{t} x^{*}(s) d s \quad \forall \gamma \in S C_{b}^{*}\left(\mathbb{R}_{+}\right)$.

ЗАмЕчАниЕ 3 . Конструкция Конна из [1] использует пару функций $\psi(t)=\log (1+t)$ и $k(t)=\log (1+t)$. Очевидно, что $\psi^{-1}=k^{-1}$ удовлетворяют предположениям теоремы 8. Следовательно, результат В) (i) $\Longleftrightarrow$ (ii) обобщает на произвольные пространства Марцинкевича утверждение, доказанное Конном в [1, предложение IV .2. $\beta .6]$. Результат В) (ii) $\Longleftrightarrow$ (iii) является новьм даже для $\psi=\kappa=\log (1+t)$.

\section{СПИСОК ЦИТИРОВАННОЙ ЛИТЕРАТУРЫ}

[1] Connes A. Noncommutative Geometry. San Diego: Academic Press, 1994.

[2] Dixmier J. Existence de traces non normales // C. R. Acad. Sci. Paris. Sér. A. 1966. V. 262. P. 1107-1108.

[3] Dodds P., de Pagter B., Semenov E., Sukochev F. Symmetric functionals and singular traces // Positivity. 1998. V. 2. P. 47-75.

[4] Доддс П. Г., де Пагтер Б., Седаев А. А., Семенов Е. М., Сукочев Ф. А. Сингулярные симметричные функционалы // Зап. научн. семин. ПОМИ. 2002. Т. 290. С. 42-71.

[5] Доддс П. Г., де Пагтер Б., Седаев А. А., Семенов Е. М., Сукочев Ф. А. Сингулярные симметричные функционалы и банаховы пределы с дополнительными свойствами инвариантности // Изв. АН. Сер. матем. 2003. Т. 67. №6. С. 111-136.

[6] Крейн С. Г., Петунин Ю. И., Семенов Е. М. Интерполяция линейных операторов. М.: Наука, 1978.

[7] Lorentz G. A contribution to the theory of divergent sequences // Acta Math. 1948. V. 80. P. $167-190$.

(С. Лорд, Ф. А. Сукочев) School of Informatics and Engineering,

(А. А. Седаев) Воронежский государственный архитектурно-строительный университет

E-mail: steven_lord_au@yahoo.com.au, sed@vmail.ru,

sukochev@infoeng.flinders . edu . au 